Editorial

\title{
Late effect clinic - An integral part of childhood cancer treatment
}

The age adjusted incidence rate of childhood cancer in India is 38-124 per million children per year. The most common malignancies in childhood include Leukaemia, Lymphoma and Brain tumours. ${ }^{1}$ The 5 year overall survival in most of the population based cancer registry in India is between $40-50 \%$, which is lower compared to western population. ${ }^{1}$ The reasons for lower survival include biology of the disease, financial constraint, accessibility to health care, sex discrimination and abandonment of treatment.

Of late with the treatment at specialized centres and a multidisciplinary approach, awareness of treatment and improvement in follow up strategies have resulted in improved survival. The treatment of a child with cancer would include surgery, radiation and chemotherapy. Each of the above modality has its own immediate and late complication and it combination can act synergistically. We would take care of the immediate complications during the treatment but forget the late side effects. Once a child completes his treatment it is imperative to look at the late side effects including immunization.

The common late-effects would include growth related effects, hormonal issues, infertility issues, cognitive effects, cardiac effects and second cancers. Oeffinger et al reported the health status of 10,397 survivors of childhood cancer treated from 1970 to $1987.62 \%$ had atleast one chronic health issues while $27 \%$ had a serious life threatening condition such as renal and cardiac problems. ${ }^{2}$ Survivors were more likely to have major joint replacement, congestive cardiac failure, second malignancy and cognitive problems.

The cardiac late sequelae may involve pericardium, the myocardium, the conduction system, the coronary arteries, and the valves. The association between anthracyclines and cardiotoxicity has been known since long especially when the dose exceeds $550 \mathrm{mg} / \mathrm{m}^{2}$. Pericarditis is the most common recognized side effect of radiation therapy in patients with Hodgkin lymphoma receiving mantle radiation. The pulmonary late effects are usually due to Bleomycin which can result in pulmonary fibrosis. The nitrosoureas used in bone marrow transplant can also result in pulmonary fibrosis. Radiation pneumonitis can result following radiation to mediastinum in children with Hodgkin lymphoma. Effects of radiotherapy, surgery and chemotherapy resulting in endocrine sequelae may manifest years later. Growth hormone deficiency, hypothyroidism, adrenal insufficiency are common long term issues. Renal toxicity is most commonly associated with chemotherapy. Cisplatin nephrotoxicity is cumulative when dose exceeds $1200 \mathrm{mg} / \mathrm{m}^{2}$. Alkylating drugs like Ifosfamide and Cyclophosphamide have been implicated in chronic kidney disease. CKD is recognized common late effect who have received stem cell transplant. Neurologic sequelae can be a significant cause of morbidity in survivors. Lateeffects of radiotherapy can result in cognitive issues affecting learning abilities and poor scholastic performance.

It is important to have continuous surveillance for late effects which can be done through late effect clinic. In western countries there are a number of such clinics whereas there is paucity in India. The clinic should have a multidisciplinary team comprising of Paediatrician, Oncologist, Endocrinologist, Cardiologist, Nurse and Social worker.

There has been an increased documentation of treatment related morbidity among childhood cancer survivors. Research has shown that late effects contribute to high burden of morbidity with $60 \%$ to $90 \%$ developing one or more chronic health conditions and $20 \%$ experience severe life threatening complication during adulthood. $^{2-4}$ With increasing survival, a risk based approach should be followed based on clinical, biological and genetic factors in order to reduce long term side effects. It is also necessary that the transition from childhood survivor to adult survivor is important and has to be taken care by an appropriate physician or a multidisciplinary team. The late effect clinic can also help identify survivors who could act as guardians for children on treatment and reduce abandonment.

In conclusion, it is imperative that all regional cancer centres should have a late effect clinic as a part of childhood cancer treatment.

\section{References}

1. Arora RS, Eden TO, Kapoor G. Epidemiology of childhood cancer in India. Indian J Cancer. 2009;46:264-273.

2. Oeffinger KC, Mertens AC, Sklar CA et al Chronic health conditions in adult survivors of childhood cancer. N Engl J Med. 2006;355:1572-1582.

3. Lorenzi MF, Xie L, Rogers PC, et al. Hospital-related morbidity among childhood cancer survivors in British Columbia, Canada: report of the childhood, adolescent, young adult cancer survivors (CAYACS) program. Int J Cancer. 2011;128: 1624-1631.

4. Wasilewski-Masker K, Mertens AC, Patterson B, et al. Severity of health conditions identified in a pediatric cancer survivor program. Pediatr Blood Cancer. 2010;54:976-982.

\author{
Department of Medical Oncology, Regional Cancer Centre \\ JIPMER, Puducherry 605 006, India \\ E-mail address: drbiswajitd@yahoo.com.
}

